\title{
Elucidation of glutamine lipid biosynthesis in marine bacteria reveals its importance under phosphorus deplete growth in Rhodobacteraceae
}

\author{
Alastair F. Smith ${ }^{1} \cdot$ Branko Rihtman $^{1} \cdot$ Rachel Stirrup $^{1} \cdot$ Eleonora Silvano ${ }^{1} \cdot$ Michaela A. Mausz ${ }^{1} \cdot$ David J. Scanlan $^{1} \cdot$ \\ Yin Chen (10) 1
}

Received: 30 April 2018 / Revised: 10 July 2018 / Accepted: 16 July 2018 / Published online: 14 August 2018

(c) The Author(s) 2018. This article is published with open access

\begin{abstract}
Marine microorganisms employ multiple strategies to cope with transient and persistent nutrient limitation, one of which, for alleviating phosphorus (P) stress, is to substitute membrane glycerophospholipids with non-P containing surrogate lipids. Such a membrane lipid remodelling strategy enables the most abundant marine phytoplankton and heterotrophic bacteria to adapt successfully to nutrient scarcity in marine surface waters. An important group of non-P lipids, the aminolipids which lack a diacylglycerol backbone, are poorly studied in marine microbes. Here, using a combination of genetic, lipidomics and metagenomics approaches, we reveal for the first time the genes $(g l s B$, ols $A)$ required for the formation of the glutaminecontaining aminolipid. Construction of a knockout mutant in either $g l s B$ or olsA in the model marine bacterium Ruegeria pomeroyi DSS-3 completely abolished glutamine lipid production. Moreover, both mutants showed a considerable growth cost under P-deplete conditions and the olsA mutant, that is unable to produce the glutamine and ornithine aminolipids, ceased to grow under P-deplete conditions. Analysis of sequenced microbial genomes show that $g l s B$ is primarily confined to the Rhodobacteraceae family, which includes the ecologically important marine Roseobacter clade that are key players in the marine sulphur and nitrogen cycles. Analysis of the genes involved in glutamine lipid biosynthesis in the Tara ocean metagenome dataset revealed the global occurrence of $g l s B$ in marine surface waters and a positive correlation between $g l s B$ abundance and $\mathrm{N}^{*}$ (a measure of the deviation from the canonical Redfield ratio), suggesting glutamine lipid plays an important role in the adaptation of marine Rhodobacteraceae to P limitation.
\end{abstract}

\section{Introduction}

Bacterial membranes form the barrier separating bacteria from their surrounding environment, with membrane lipids being an essential component of this structure. Our knowledge of bacterial lipids is predominantly derived from studies of model organisms, e.g., Escherichia coli, which is primarily composed of several glycerophospholipids, phosphatidylethanolamine, phosphatidylglycerol and a small amount of

Electronic supplementary material The online version of this article (https://doi.org/10.1038/s41396-018-0249-z) contains supplementary material, which is available to authorized users.

Yin Chen

y.chen.25@warwick.ac.uk

1 School of Life Sciences, University of Warwick, Coventry CV4 7AL, UK cardiolipin [1]. However, beyond Escherichia coli, we now know that a range of lipids are found in bacterial membranes, including phosphorus (P)-containing glycerophospholipids but also P-free lipids that are composed of a diacylglycerol backbone. The latter include betaine lipids, e.g., diacylglycerol- $N, N, N$-trimethylhomoserine (DGTS), sulfolipids, e.g., sulfoquinovosyl-diacylglycerol and glycolipids, e.g., monoglycosyl-diacylglycerol (MGDG) and glucuronic acid diacylglycerol (GADG) [2]. In the marine environment, it is well established that $\mathrm{P}$ availability significantly affects lipid composition in marine phytoplankton as well as cosmopolitan marine heterotrophic bacteria [3-5]. In fact, several lipid surveys (environmental lipidomics) have been carried out in marine waters and the ratio of non-P lipids to phospholipids is a useful marker for detecting P-stress in natural microbial communities (e.g., [3, 4, 6-8]).

An important, yet poorly studied group of P-free lipids are the amino-acid containing lipids [9]. Unlike the aforementioned lipids, these aminolipids do not contain a diacylglycerol 
backbone. Instead, these aminolipids contain an amino acid head group linked to a fatty acid (usually a $\beta$-hydroxy fatty acid) through an amide bond. Arguably, the best studied aminolipid is the ornithine lipid which contains the nonproteinogenic amino acid ornithine as the head group. Ornithine lipids have been widely reported in bacteria [10], being found in marine surface water lipidomic surveys [6] and the abundant marine heterotroph SAR11 [5]. Biosynthesis of ornithine lipids is carried out either by a two-step process using two acyltransferases encoded by the ols $B$ and ols $A$ genes or by the bifunctional fusion protein OlsF [11]. Other amino acid head groups found in bacterial aminolipids include glutamine, lysine and serine [9, 12]. However, these aminolipids have not been reported in environmental lipidomics surveys of marine waters and the metabolic pathways underpinning the biosynthesis of non-ornithine aminolipids are unknown.

Here, we report the identification and characterisation of glutamine lipid in members of the cosmopolitan marine Roseobacter clade, a group of Alphaproteobacteria that are abundant in coastal marine waters and play important roles in the biogeochemical cycling of $\mathrm{S}$ and $\mathrm{N}$ (see reviews by [13, 14] and references therein). We reveal the genes required for glutamine lipid biosynthesis and demonstrate that this glutamine lipid is predominantly found in marine Roseobacter and closely related members of the Rhodobacteraceae family. Moreover, this lipid appears to be important for maintaining normal cellular function during $\mathrm{P}$ deplete conditions.

\section{Materials and methods}

\section{Bacterial strains, media and cultivation conditions}

Bacterial strains, plasmids and PCR primers used in this study are listed in Suppl. Table S2. Marine bacteria used in this study were cultivated using either marine broth (Difco Marine Broth 2216 (Becton, Dickinson and Company, Sparks, MD, USA), $1 / 2$ YTSS ( $2 \mathrm{~g} / \mathrm{L}$ yeast extract, $1.25 \mathrm{~g} / \mathrm{L}$ peptone, $20 \mathrm{~g} / \mathrm{L}$ sea salts, Sigma-Aldrich), or a defined MAMS medium [15]. E. coli strains were routinely cultivated in lysogeny broth with appropriate antibiotics.

\section{Construction of mutants of Ruegeria pomeroyi}

Marker-exchange mutagenesis was used to construct the $\Delta g l s B$ (SPO2489) and $\Delta$ olsA (SPO1980) mutants of $R$. pomeroyi DSS-3 [16]. Briefly, primers were designed to PCR amplify 500-700 base pair regions either side of the target gene. These two fragments, together with a gentamicin-resistant cassette, were cloned into vector

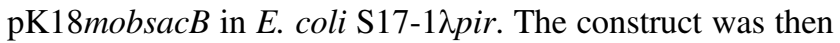
conjugated to $R$. pomeroyi DSS-3. Transformants were selected on marine sea salt agar plates supplemented with
$10 \mathrm{mM}$ glucose, $2 \mathrm{mM}$ glycine betaine and $10 \mu \mathrm{g} / \mathrm{mL}$ gentamicin. Double-crossover deletion mutants were selected for their sensitivity to kanamycin $(50 \mu \mathrm{g} / \mathrm{mL})$. The mutants were confirmed by PCR and subsequent sequencing.

To compare the growth of the $\Delta g l_{s} B$ and $\Delta o l s A$ mutants with wild-type $R$. pomeroyi DSS-3, cells were grown in defined MAMS medium with either $0.5 \mathrm{mM}$ or $5 \mathrm{mM}$ phosphate in three biological replicates. Bacterial growth was quantified by measuring optical density (OD) at 540 $\mathrm{nM}$ at regular intervals. Alkaline phosphatase activity was measured prior to the collection of samples for lipid analysis. Pairwise comparisons of the growth rates of each strain grown at high and low phosphate concentrations, as well as comparisons of the growth rates between strains grown at the same phosphate concentration, were made using a Student's $t$-test.

\section{Intact polar lipid extraction and analysis}

Lipids from bacterial cultures were extracted using a modified Folch extraction method [17]. Briefly, $1 \mathrm{~mL}$ of culture with an $\mathrm{OD}_{540} \sim 0.5$ was collected. The cells were pelleted by centrifugation and re-suspended in $0.5 \mathrm{~mL}$ of LC-MS grade methanol (Sigma-Aldrich) in a $2 \mathrm{~mL}$ glass Chromacol vial (Thermo Scientific). Lipid extraction was carried out using chloroform-methanol. Solvent-extracted lipids were dried under nitrogen gas using a Techne sample concentrator (Staffordshire, UK) and lipid pellets were re-suspended in

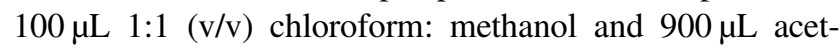
onitrile. These samples were then analysed by LC-MS employing a Dionex 3400RS HPLC, coupled to an AmazolSL quadrupole ion trap MS (Bruker Scientific) via an electrospray ionisation interface. Separation of lipids in HPLC was carried out using a BEH amide XP column (Waters). The column was maintained at $30{ }^{\circ} \mathrm{C}$, with a flow rate of $150 \mu \mathrm{L} \mathrm{min}{ }^{-1}$. Samples were run on a 15 -min gradient from $95 \%(\mathrm{v} / \mathrm{v})$ acetonitrile to $28 \%$ (w/v) $10 \mathrm{mM}$ ammonium acetate $\mathrm{pH} 9.2$, with 10 minutes equilibration between samples. Each sample was analysed in both positive and negative ionisation modes. Data analyses were carried out using the Bruker Compass software package, using DataAnalysis for peak identification and characterization of lipid class, and QuantAnalysis for quantification of the relative abundance of aminolipids to phosphatidylethanolamine.

\section{Alkaline phosphatase activity assay}

Alkaline phosphatase activity was used to assess whether cultures were stressed for $\mathrm{P}$ availability using paranitrophenol phosphate (pNPP) as the substrate. A stock solution of $10 \mathrm{mM}$ pNPP (Sigma-Aldrich) was prepared in $10 \mathrm{mM}$ Tris- $\mathrm{HCl} \mathrm{pH}$ 7.0. $900 \mu \mathrm{L}$ aliquots of cell culture 
were added to $100 \mu \mathrm{L}$ pNPP stock solution to obtain a final pNPP concentration of $1 \mathrm{mM}$. Control incubations were set up without the cultures in parallel. Formation of the yellowcoloured para-nitrophenol (pNP) was recorded by monitoring absorbance at $405 \mathrm{nM}$ using a BioRad imark microplate reader. A calibration curve was made using pNP standards (Sigma-Aldrich) in the range between $10 \mu \mathrm{M}-2$ $\mathrm{mM}$.

\section{Phylogenetic and metagenome/metatranscriptome analyses}

Phylogenetic analysis of 16S rRNA genes from Rhodobacteraceae was carried out using the full length 16S rRNA gene retrieved from the IMG database (https://img.jgi.doe. gov/). GlsB and OlsB sequences were retrieved from the IMG database using BLASTP searches using SPO2489 and SPO1980 as the query sequence, respectively, with an $e$ value cut-off of $10^{-5}$. The retrieved homologues were then manually inspected using the neighbourhood view in IMG for the presence of bamE and olsA, in the neighbourhood of $g l s B$ and $o l s B$, respectively. Sequence alignment was performed using Muscle and phylogenetic analyses were performed with RaxML with 100 bootstrap replicates [18].

To search for GlsB, OlsB and OlsF homologues in the Tara metagenome datasets, a single Hidden Markov model profile was constructed using Hmmer3 with an $e$-value cutoff of $10^{-5}$. The reference sequence was chosen to represent $\mathrm{OlsB}, \mathrm{GlsB}$ and OlsF whose functions had been validated experimentally. This reference alignment was used to construct a maximum likelihood phylogenetic tree using RaxML with 100 bootstrap replicates. In order to classify the sequences retrieved from the Tara metagenomes by Hmm search, their maximum likelihood placement onto this reference phylogeny was determined using pplacer [19]. The abundance of each gene in the Tara metagenomes was standardised to RecA abundance retrieved using an $\mathrm{Hmm}$ search using the same $e$-value cut-off.

To search for $g l s B$ in metatranscriptome datasets, we used the JGI IMG metatranscriptome database, which contained 428 datasets from marine ecosystems (as of 12 June 2018). A BlastP search was carried out using GlsB from Ruegeria pomeroyi DSS-3 as the query sequence (SPO2489) with a stringent $e$-value cut-off of $10^{-20}$, which yielded 131 sequences at varying lengths (Table S1). The taxonomy of the retrieved GlsB sequences was assigned by a BlastP search against the NCBI non-redundant protein sequences and the top BlastP hit, together with sequence similarity value, $e$-value and accession number, is presented in Table S1. GlsB sequences retrieved from the metatranscriptome datasets ( $>140$ amino acids in length) were then aligned and mapped to the $\mathrm{GlsB} / \mathrm{OlsB} / \mathrm{OlsF}$ reference tree to confirm their phylogenetic position (Figure S3).

\section{Statistical analysis}

Linear regression was used to investigate relationships between the abundance of $o l s B, g l s B$ and $o l s F$ genes and a measure of relative phosphate availability in the Tara metagenome dataset. Since many samples in the Tara metagenome dataset have a very low phosphate concentration, the ratio of nitrogen-to-phosphorus traditionally used in microbial ecology is problematic as the denominator is close to zero. Therefore, we chose to use the measure of the relative abundance of nitrogen-to-phosphorus introduced by Weber and Deutsch [20] where $N^{*}=\left[\mathrm{NO}_{3}^{-}\right]-16\left[\mathrm{PO}_{4}^{3-}\right]$. The abundance of $\mathrm{ols} B, \mathrm{gls} B$ and ols $F$ takes the form of the aforementioned count data retrieved from each Tara metagenome. In order to assess whether $\mathrm{N}^{*}$ was a significant predictor of the abundance of each aminolipid synthesis gene (i.e., ols $B, g l s B$ and $o l s F$ ), two models were compared for each gene. A base model was constructed in which the abundances of each of the microbial groups to which sequences for that gene were assigned were included as covariates (Suppl. Table S3). This was compared to the second model that was identical to the base model but with the addition of a term for $\mathrm{N}^{*}$ using likelihood ratio tests. Scatterplots and generalised linear model fits showing the relationship between aminolipid synthesis gene counts and $\mathrm{N}^{*}$ in the Tara metagenomes are shown in Suppl. Figure S4. The abundance values for each microbial group were calculated from metagenomic 16S Illumina tag data, which is available from http:// ocean-microbiome.embl.de/companion.html [21, 22].

\section{Results}

\section{Identification of glutamine lipids in Ruegeria pomeroyi DSS-3 by mass spectrometry}

We have previously grown several marine Roseobacter strains in the laboratory in order to investigate the link between nutrient availability and lipid remodelling in these ecologically important marine bacteria [3]. When analysed by high-performance liquid chromatography (HPLC)-mass spectrometry (MS) in negative ionisation mode, these strains revealed the presence of a new lipid that consistently eluted at $\sim 9.6 \mathrm{~min}$ in several Roseobacter strains tested, including Ruegeria pomeroyi DSS-3 (Fig. 1). The most abundant lipid species at $\sim 9.6 \mathrm{~min}$ has a mass to charge ratio $(\mathrm{m} / \mathrm{z})$ of 717 , corresponding to one of the most abundant lipid species previously observed in Rhodobacter sphaeroides [23]. In order to investigate the structure of this $\mathrm{m} / \mathrm{z}=$ 717 ion, multiple rounds of fragmentation $\left(\mathrm{MS}^{\mathrm{n}}\right)$ were performed using a quadrupole ion trap MS and the sequential fragmentation patterns obtained (Fig. 1). These 
a)

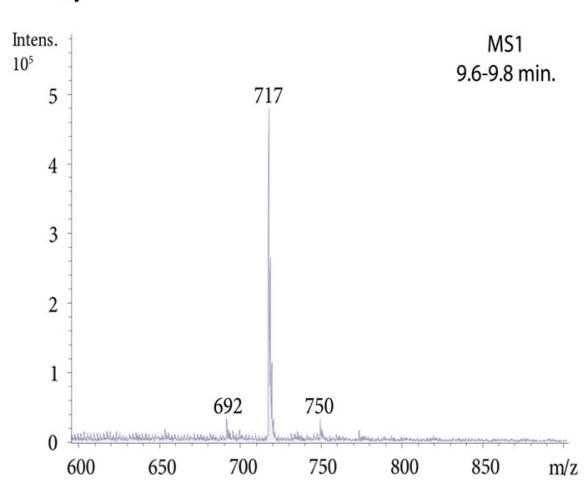

b)

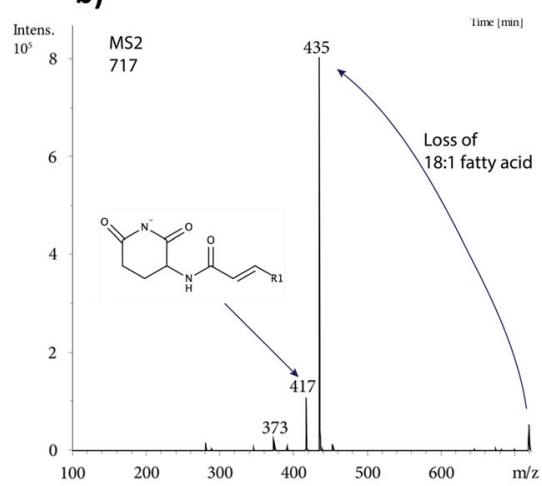

c)

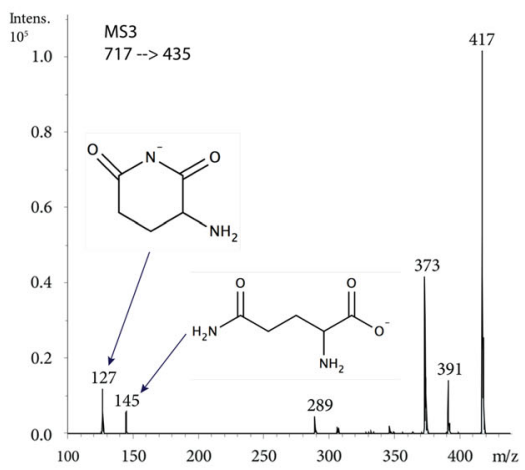

Fig. 1 Characterisation of glutamine lipid from Ruegeria pomeroyi DSS-3 cultures by mass spectrometry in negative ion mode. a Mass spectrum showing molecular ions detected in the peak eluting between 9.6 and 9.8 minutes. The most abundant ion, with $m / z$ 717, was selected for further fragmentation $(\mathbf{b}, \mathbf{c})$. After a first round $\left(\mathrm{MS}^{2}\right)$ of fragmentation $(\mathbf{b})$, the major ion with $\mathrm{m} / \mathrm{z} 435$ was consistent with a loss of an $18: 1$ fatty acid. This ion was selected for $\mathrm{MS}^{3}$ fragmentation (c), which yielded diagnostic ions with $\mathrm{m} / \mathrm{z} 145$ and 127 , corresponding to glutamate and to an ion resulting from the cyclisation of glutamate following loss of water, respectively

patterns are consistent with the presence of a glutamine head group [23], indicated by two characteristic ions with $\mathrm{m} / \mathrm{z} 145$ and 127, respectively, which corresponds to glutamine after the loss of a proton and cyclisation of glutamate following loss of a water molecule (Fig. 1).

\section{The SPO2489 gene is required for glutamine lipid biosynthesis in Ruegeria pomeroyi DSS-3}

Having confirmed the presence of glutamine lipid in $R$. pomeroyi DSS-3, we set out to identify the genes involved in its biosynthesis. To the best of our knowledge the presence of this aminolipid has only been previously reported in Rhodobacter sphaeroides [12, 23]. However, the genes underpinning glutamine lipid biosynthesis are unknown. Due to its structural similarity to ornithine lipid, which is probably the best studied bacterial aminolipid, it has been previously hypothesised that an $N$ acetyltransferase is required for the initial condensation of glutamine to a 3-hydroxy fatty acid, followed by an $O$ acetyltransferase for adding a second fatty acid [9]. In Rhodobacter sphaeroides and Ensifer meliloti, the twostep ornithine lipid biosynthesis pathway is carried out by $o l s B$ and $o l s A$, encoding an $N$-acetyltransferase and an $O$ acetyltransferase, respectively (Fig. 2a). Close investigation of the $R$. pomeroyi DSS-3 genome allowed the identification of OlsB and OlsA, encoded by SPO1980 and SPO1979, respectively (Fig. 2b). Interestingly a second olsB-like gene (SPO2489) was also found in the $R$. pomeroyi DSS-3 genome, showing $29 \%$ sequence identity to OlsB. We therefore speculated that SPO2489 (hereafter designated as $g l s B$ for glutamine lipid synthesis) is involved in glutamine lipid biosynthesis. Because no other OlsA-like $O$-acetyltransferase was found in the $R$. pomeroyi DSS-3 genome, we suspected olsA was also responsible for glutamine lipid synthesis (Fig. 2a).
To test the hypothesis that $g l s B$ and $o l s A$ are involved in glutamine lipid biosynthesis, we constructed marker exchange mutants using a gentamicin resistance cassette in $R$. pomeroyi DSS-3 [3, 16]. As predicted, a deletion mutant in either $g l s B$ or ols $A$ completely abolished the formation of the glutamine lipid, as assessed by MS of membrane lipid extracts (Fig. 2c). However, whilst deletion of $g l s B$ did not affect the formation of ornithine lipid (data not shown), the ols $A$ mutant was also unable to synthesise ornithine lipid, agreeing with our proposed biosynthetic pathway model (Fig. 2a) that ols $A$ is responsible for the last step of both ornithine and glutamine lipid biosynthesis in this bacterium.

\section{Characterization of glutamine lipid mutants under phosphorus stress}

In order to investigate the role of the glutamine lipid in $R$. pomeroyi DSS-3 in response to $\mathrm{P}$ availability, we used a defined marine ammonium mineral salts (MAMS) medium and compared the growth of the wild-type, $\Delta o l s A$ and $\Delta g l s B$ mutants, the latter two strains being unable to synthesize this glutamine lipid. A concentration of $0.5 \mathrm{mM}$ phosphate was sufficient to induce $\mathrm{P}$ stress in this bacterium, with alkaline phosphatase activity in the wild-type in these low $\mathrm{P}$ grown cultures $\left(6.25 \pm 0.97 \mu \mathrm{M} \mathrm{pNP} \mathrm{h}^{-1} \mathrm{OD}_{540}{ }^{-1}\right)$ significantly higher ( $t$-test, $p<0.001)$ than wild-type cells grown in high $\mathrm{P}$ medium $(5 \mathrm{mM})\left(0.86 \pm 0.09 \mu \mathrm{M} \mathrm{pNP} \mathrm{h}^{-1}\right.$ $\left.\mathrm{OD}_{540}{ }^{-1}\right)$. When the $\Delta o l s A$ and $\Delta g l s B$ mutants were cultivated in high $\mathrm{P}$ medium, no significant difference in growth rate was observed (Table 1). However, when the mutants were cultivated in low $\mathrm{P}$ medium, the $\Delta o l s A$ mutant failed to grow (Suppl. Figure S1a) and the $\Delta g l s B$ mutant had a significantly reduced growth rate $\left(0.077 \pm 0.012 \mathrm{~h}^{-1}\right)$ compared to that of the wild-type $\left(0.096 \pm 0.008 \mathrm{~h}^{-1}\right)$.

We further analysed the lipidome of wild-type Ruegeria pomeroyi DSS-3 and the $\Delta g l s B$ mutant under high $(5 \mathrm{mM})$ 
a)<smiles>NCCCC(N)C(=O)O</smiles><smiles>C1CCCC1</smiles><smiles>CC(O)CC(=O)NC(C)CCCN</smiles>

Lyso-ornithine lipid<smiles>CCCC</smiles><smiles>CCCCC(C)NC(=O)CC(C)OC(N)=O</smiles><smiles>CC(=O)CCC(C)C</smiles><smiles>CC(O)CC(=O)O</smiles><smiles>CC(C)C</smiles><smiles>CC(=O)CCC(C)NC(=O)CC(C)O</smiles>

b)

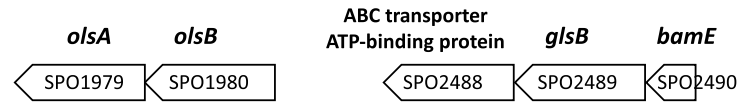

c)

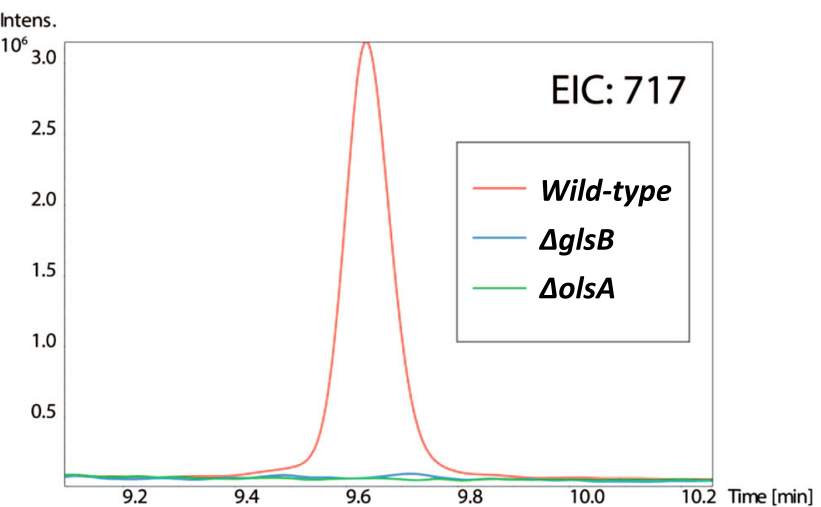

Fig. 2 a Proposed pathway for the biosynthesis of glutamine lipid, in comparison to ornithine lipid biosynthesis, in R. pomeroyi DSS-3. The first step is carried out by an $N$-acetyltransferase encoded by $g l_{s} B$ and $o l s B$, respectively and lysolipid intermediates are formed. The second step is mediated by an $O$-acetyltransferase encoded by olsA by esterification of a second fatty acid to the hydroxyl group of the lysolipid intermediates. b The gene neighbourhood of olsB (SPO1980) and glsB (SPO2489) in the genome of $R$. pomeroyi DSS-3. c Extracted ion chromatograms (EIC) obtained after analysing lipid extract from the wild-type $R$. pomeroyi DSS-3 and the olsA and glsB mutants by mass spectrometry in negative ion mode. Ions with mass-to-charge $(\mathrm{m} / \mathrm{z}) 717$ correspond to the intact mass of the glutamine lipid (Fig. 1)

Table 1 Growth rates of the glutamine lipid mutants compared with the wild-type Ruegeria pomeroyi DSS-3 at different P concentrations in a defined minimal medium

\begin{tabular}{lll}
\hline & \multicolumn{2}{l}{ Growth rate $\left(\mathrm{h}^{-1}\right)$} \\
\cline { 2 - 3 } & $5 \mathrm{mM}$ phosphate & $0.5 \mathrm{mM}$ phosphate \\
\hline Wild-type & $0.110 \pm 0.008$ & $0.096 \pm 0.008$ \\
$\Delta$ gls $B$ mutant & $0.108 \pm 0.005$ & $0.077 \pm 0.012$ \\
$\Delta$ ols $A$ mutant & $0.096 \pm 0.012$ & No growth \\
\hline
\end{tabular}

and low phosphate $(0.5 \mathrm{mM})$ conditions. Because the $\Delta$ ols $A$ mutant produced neither the glutamine nor ornithine lipid and failed to grow in low phosphate medium, its lipidome was not analysed further. Ruegeria pomeroyi DSS-3 does not have the PlcP-mediated lipid remodelling pathway [3]. The only lipids that are made comprising glycerol-based backbones are glycerophospholipids (phosphatidylethanolamine, PE and phosphatidylglycerol, PG); DGTS, MGDG and GADG were not found. Due to the lack of available standards for aminolipids, we compared relative abundance using the ratio of glutamine lipid (QL) to $\mathrm{PE}$ and ornithine lipid (OL) to PE under high and low phosphate conditions (Fig. 3). This analysis showed that wild-type Ruegeria

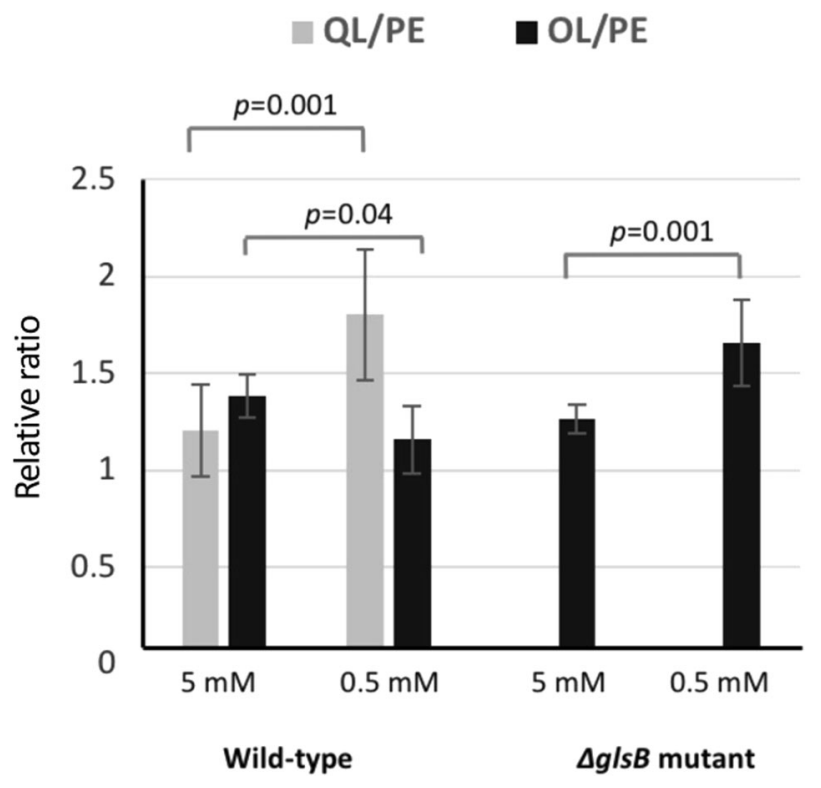

Fig. 3 The relative abundance of glutamine lipid (QL) and ornithine lipid (OL), normalised against phosphatidylethanolamine (PE) in the wild type and the $\Delta g l s B$ mutant under high $(5 \mathrm{mM})$ and low $(0.5 \mathrm{mM})$ phosphate conditions. Measurements were carried out in three biological replicates each with three technical replicates and the error bars represent standard deviation 


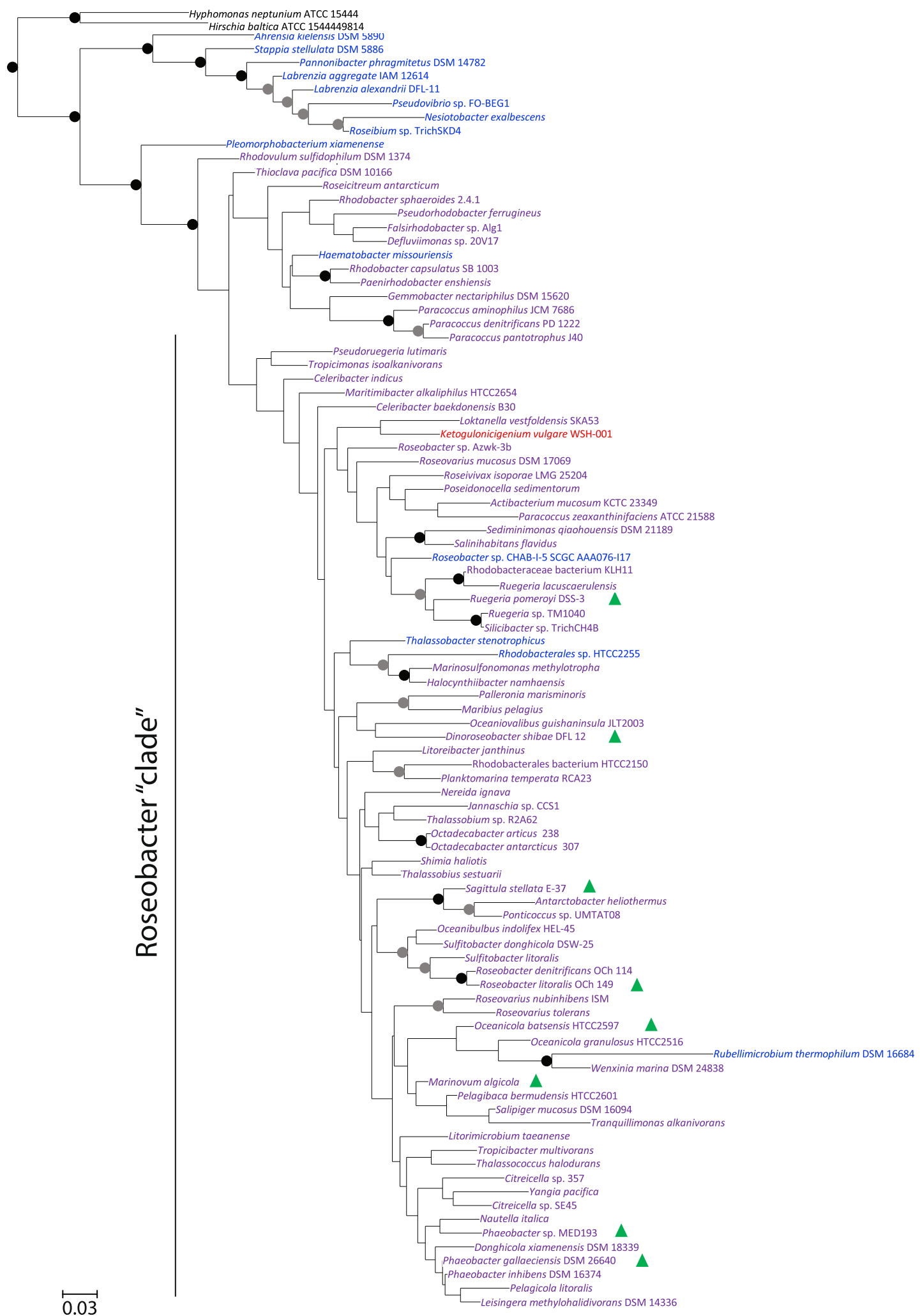

Fig. 4 Maximum likelihood 16S rRNA gene phylogeny of Rhodobacteraceae with fully or partially sequenced genomes. Bootstrap support for nodes is indicated by filled circles with a black circle indicating support $>70 \%$, a grey circle indicating $50-70 \%$ and the absence of circles indicating $<50 \%$ bootstrap support. The colours indicate the presence of ols $B$ alone (blue), glsB alone (red) or both homologues (purple) in the genome as detected by BLASTP searches using an $e$-value cut-off of $10^{-5}$. Strains that are verified for the production of glutamine lipid in this study by liquid chromatography-mass spectrometry are indicated by a green triangle 
pomeroyi DSS-3 had a significantly elevated ratio of QL:PE under $\mathrm{P}$ stress conditions ( $t$-test, $p=0.001$ ) suggesting a substitution of PE for the glutamine lipid under these conditions. On the other hand, the OL:PE ratio did not change under $\mathrm{P}$ stress conditions in wild type Ruegeria pomeroyi DSS-3. However, in the $\Delta g l s B$ mutant, which does not produce glutamine lipid, the OL:PE ratio significantly increased under P stress. Quantification of the change in PE in wild-type Ruegeria pomeroyi DSS-3 and the $\Delta g l s B$ mutant under high and low $\mathrm{P}$ conditions (Suppl. Figure $\mathrm{S} 1 \mathrm{~b}$ ) showed PE levels were significantly reduced under low $\mathrm{P}$ conditions. Taken together, our data suggests that the glutamine lipid is important for $R$. pomeroyi DSS-3 to maintain maximal cell growth particularly during $\mathrm{P}$ stress conditions and that the glutamine and ornithine lipids may be functionally interchangeable in this bacterium.

\section{glsB gene presence appears to be restricted to the Rhodobacteraceae family}

Since the presence of the glutamine lipid has only previously been reported in Rhodobacter sphaeroides [12, 23], we set out to investigate the distribution of glutamine lipid biosynthesis potential in genome-sequenced bacteria in the integrated microbial genomes (IMG) database using $g l s B$ as the functional gene marker. Interestingly, this analysis indicated that $g l s B$ is only found in bacteria of the Rhodobacteraceae family. In contrast, the olsB/ols $F$ genes are more widespread across bacterial phyla, including Proteobacteria and Bacteroidetes, agreeing with a previous study showing that around half of the genome-sequenced bacteria are capable of producing ornithine lipids [9]. Notably, glsB occurs widely in the Rhodobacter-Paracoccus group as well as the marine Roseobacter clade (Fig. 4), including true pelagic Roseobacter strains such as Planktomarina temperata RCA23 [24] and Rhodobacterales sp. HTCC2150 [25]. The Rhodobacter-Paracoccus group and the marine Roseobacter group are evolutionally related and may come from a common ancestor according to a recent phylogenomics analysis [26]. To confirm the occurrence of glutamine lipids in the Rhodobacteraceae we extracted membrane lipids from selected strains of the Roseobacter group and analysed the presence of the $m / z 717$ ion by mass spectrometry. We indeed found glutamine lipids present in all the cultures analysed (Fig. 4).

\section{Glutamine lipid biosynthesis in marine metagenomes and metatranscriptomes}

In order to better understand the role of glutamine lipids in marine ecosystems, we next investigated the distribution of $g l s B$ (for glutamine lipid synthesis) and olsB/olsF (for ornithine lipid synthesis) in the Tara ocean metagenome data set
[27]. Because GlsB and OlsB/OlsF shows significant amino acid sequence similarity, we first verified whether genes responsible for ornithine and glutamine lipid biosynthesis can be reliably separated phylogenetically using sequences from the Rhodobacteraceae genomes. This analysis showed that GlsB/OlsB sequences were consistently separated into two major clades (100\% bootstrap support) and that these two clades were congruent with a classification based on synteny (Suppl. Figure S2). The olsB gene is found in the neighbourhood of olsA whereas glsB is located next to the bamE gene which encodes a membrane lipoprotein involved in outer membrane protein assembly [28].

To classify environmental sequences retrieved from the Tara metagenomes using this phylogenetic approach, an alignment of reference sequences of OlsB, GlsB and the $N$ acyltransferase domain of OlsF was created (Fig. 5a). The functions of these genes have either been verified experimentally (see above, $[5,11]$ ) or, in the case of marine strains, the strain has been shown to produce ornithine lipid or glutamine lipid. An HMMER profile was built and used as a query to search the Tara metagenomes ( $e$-value cut-off $10^{-5}$ ). This analysis retrieved 5,097 sequences, 567 of which are classified as $g l s B$. Genes encoding ornithine lipid biosynthesis are more abundant than $g l s B$ in these metagenomes (Fig. 5b). Phylogenetically, the majority (85\%) of $g l s B$ genes are classified within the Rhodobacteraceae family in agreement with the predominant occurrence of $g l s B$ in genome-sequenced isolates of the Rhodobacteraceae family. However, the relative abundance of $g l s B$, normalised against the abundance of the single copy recA gene, did not show an obvious distribution pattern across Tara metagenome sampling sites (Fig. 5c).

In order to disentangle the multiple sources of variation likely to be driving the distribution of $g l s B$ in Tara ocean metagenomes, we employed a linear regression model [20] to test the hypothesis that genes involved in aminolipid biosynthesis, including $g l s B$, are more abundant in P-depleted areas of the ocean. Data presented in Table 2 show a significant correlation between $g l s B$ and $N^{*}$

Table 2 Likelihood ratio test (LRT) comparisons of generalised linear models for aminolipid synthesis gene abundance with and without the inclusion of $\mathrm{N}^{*}$ as an independent variable

\begin{tabular}{llllllll}
\hline & & & & \multicolumn{3}{l}{$\begin{array}{l}\text { Model } \\
\text { comparison }\end{array}$} \\
\cline { 5 - 8 } Gene & $\begin{array}{l}\text { Slope } \\
\text { coefficient }\end{array}$ & $\begin{array}{l}\text { Standard } \\
\text { error }\end{array}$ & z-value & $p$ & $\begin{array}{l}\text { LRT } \\
\text { statistic }\end{array}$ & LRT $p$ \\
\hline glsB & 0.179 & 0.087 & 2.06 & $<0.05$ & 4.35 & $<0.05$ \\
olsB & 0.201 & 0.056 & 3.62 & $<0.001$ & 13.2 & $<0.001$ \\
olsF & -0.078 & 0.077 & -1.03 & 0.304 & 1.22 & 0.269 \\
\hline
\end{tabular}

Coefficients are given for the $\mathrm{N}^{*}$ term along with $z$-value and associated $p$-values for the inclusion of $\mathrm{N}^{*}$ as a parameter. $\mathrm{N}^{*}$ is defined as $\left[\mathrm{NO}_{3}^{-}\right]-16\left[\mathrm{PO}_{4}^{3-}\right]$ 
a)

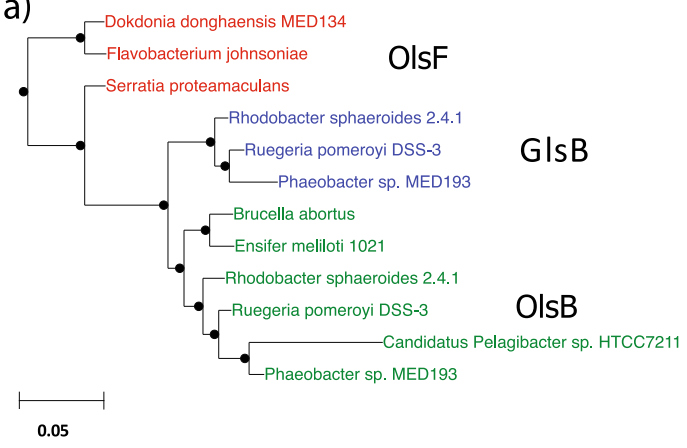

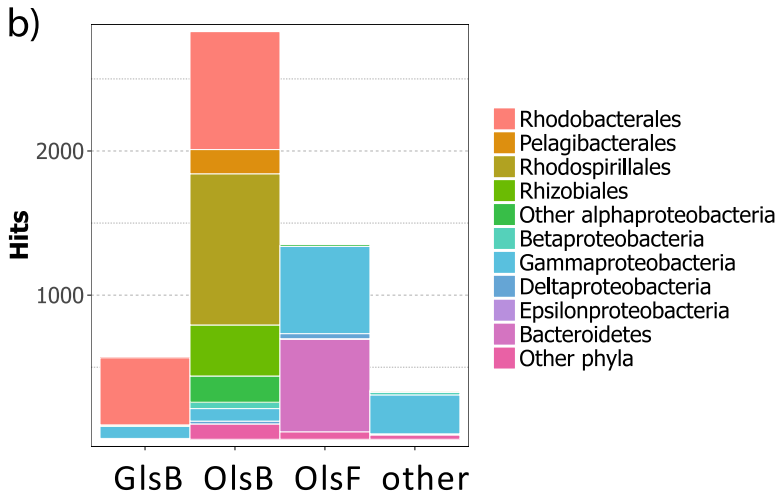

c)

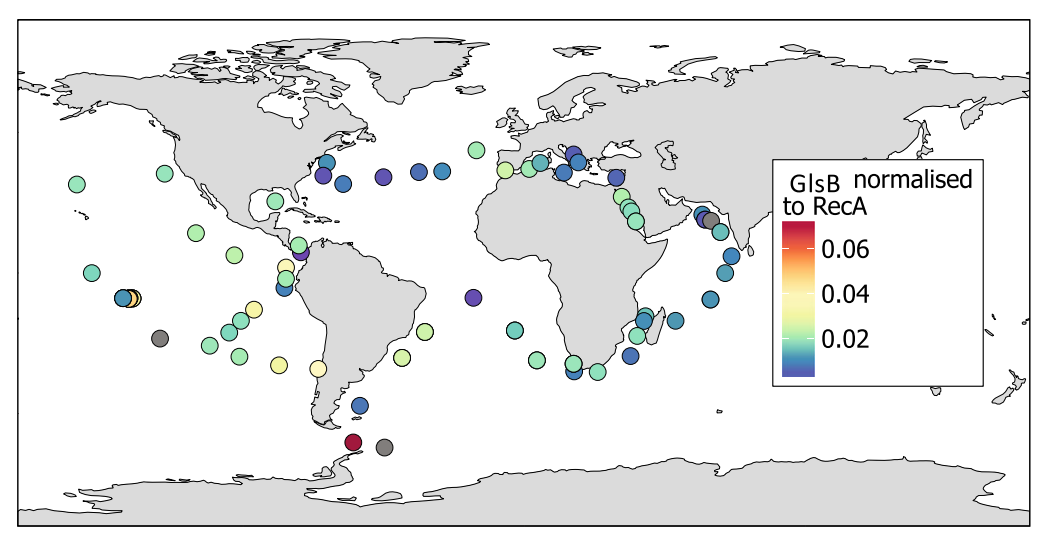

Fig. 5 a Maximum likelihood phylogeny showing the evolutionary relationship between $N$-acyltransferases involved in glutamine lipid (GlsB) and ornithine lipid biosynthesis (OlsB and $\mathrm{OlsF})$. b The number of sequences retrieved from the Tara metagenome data set that are assigned to each gene. Other represents environmental sequences which could not be unambiguously classified using our phylogenetic approach. $\mathbf{c}$ Global maps of the abundance of GlsB, normalised to the abundance of RecA in the Tara metagenome dataset. Only surface water samples (collected at $5 \mathrm{~m}$ depth) are shown. Grey circles indicate no sequences corresponding to that gene were detected in the sample

$\left(\left[\mathrm{NO}_{3}^{-}\right]-16\left[\mathrm{PO}_{4}^{3-}\right]\right)$, an indicator of the relative concentration of inorganic $\mathrm{N}$ and $\mathrm{P}$; [20]) as well as olsB and $\mathrm{N}^{*}(p<0.05$ and $p<0.001$, respectively), suggesting that the relative abundance of these two genes are indeed positively correlated to the relative concentration of the inorganic nutrients $\mathrm{N}$ and $\mathrm{P}$. In contrast, ols $F$ showed no significant correlation to the relative concentration of the inorganic nutrients $\mathrm{N}$ and $\mathrm{P}$. A close investigation of the $o l s B-\mathrm{N}^{*}$ relationship indicates that the greater slope coefficient in $o l s B$, compared to $g l s B$, is likely driven by the presence of SAR11 genes in the ols $B$ dataset but not in the $g l s B$ dataset since SAR11 isolates are known to produce ornithine lipids but not glutamine lipids in response to P-depletion [5].

Together, analysis of the genes involved in glutamine lipid and ornithine lipid biosynthesis in these Tara ocean metagenomes suggest these lipids are important in adapting to nutrient stress in abundant marine bacteria, especially the Rhodobacteraceae.

We next determined if the $g l s B$ gene is indeed actively expressed in the marine environment. Searching available metatranscriptomes in the JGI IMG database, using a stringent $e$-value cut-off of $10^{-20}$, we retrieved more than
100 hits, the majority of which (>95\%) are classified as Rhodobacteraceae (Table S1). Phylogenetic analysis showed that the actively expressed $g l s B$ genes largely originated from pelagic Roseobacter strains (Figure S3), e.g. Planktomarina temperata RCA23 [24], Rhodobacteraceae sp. HIMB11 [29], and Rhodobacteraceae sp. SB2 [30].

\section{Discussion}

Aminolipids are a poorly studied class of lipids, which seem to be found exclusively in bacteria [9]. Although several aminolipids have been identified in bacteria, only the biosynthesis of ornithine lipid has been characterised previously $[11,31,32]$. In this study, using the marine bacterium $R$. pomeroyi DSS-3 as a model, we characterized the $g l s B$ gene responsible for the first step in glutaminecontaining aminolipid formation. A second gene, olsA, which has previously been shown to convert lyso-ornithine to ornithine lipid [32], was also required for glutamine lipid biosynthesis (Fig. 2). These findings indicate that glutamine lipid biosynthesis likely proceeds via a two-step process, analogous to the synthesis of ornithine lipid (Fig. 2). The 
first step in glutamine lipid biosynthesis, the $N$-acylation of glutamine with a 3-hydroxy fatty acid is mediated by GlsB. The second step, the $O$-acylation of the hydroxyl group of the first fatty acid, appears to be catalysed by the acyltransferase OlsA. OlsA can also acylate glycerol-3phosphate to form phosphatidic acid, an intermediate in phospholipid biosynthesis [33], indicating that it has a relatively broad substrate specificity. Interestingly, lipidomics analyses of the $R$. pomeroyi mutants showed that disruption of ols $A$ did not result in an accumulation of lysoaminolipids, which might be expected to accumulate based on the proposed biosynthetic pathway (Fig. 2). However, this lack of detectable lyso-aminolipids is consistent with prior studies in E. meliloti $\Delta$ olsA mutant strains [34]. It would appear that these lyso-aminolipids are under tight control in the cell and rapidly degraded if they are not acylated by OlsA to form the intact aminolipid.

The specific physiological role of glutamine lipid in Roseobacters remains unclear. A slight growth defect was observed for the $\Delta g l s B$ mutant of $R$. pomeroyi DSS-3, deficient in glutamine lipid but not ornithine lipid biosynthesis, in low-P medium relative to the wild type (Table 1). Interestingly, the $\Delta$ ols $A$ mutant, deficient in both glutamine lipid and ornithine lipids, exhibited a more severe growth phenotype in high-P medium and ceased to grow in low-P medium (Table 1). Our data therefore strongly suggest that these aminolipids are required for normal cell function, particularly during P-deplete growth. It is likely that ornithine lipids and glutamine lipids may functionally substitute for one another, resulting in a more severe phenotype when both are removed. Repeated attempts to grow the $\Delta o l s A$ mutant in a range of phosphate concentrations below $0.5 \mathrm{mM}(50 \mu \mathrm{M}-0.25 \mathrm{mM})$ reproduced this lack of growth (data not shown). The reason for this lack of viability in low-P medium is unclear: one explanation could be that a sufficient concentration of phosphate ions is required to stabilise the membrane in the absence of either aminolipid in the $\Delta$ ols $A$ mutant. This would be analogous to the phenotype of $E$. coli mutants lacking phosphatidylethanolamine, which require divalent cations (such as $\mathrm{Ca}^{2+}$ ) for viability [35].

In contrast, the role of ornithine lipid in bacterial physiology has been studied in several model bacteria (reviewed by [10] and references therein). Previous findings of a role for this lipid in maintaining optimal amounts of $c$ type cytochromes in Rhodobacter capsulatus supports the view of aminolipids playing an integral role in Rhodobacteraceae biology [36]. However, in E. meliloti, a lack of ornithine lipids had a minimal impact on fitness except when $\mathrm{P}$ was limiting [34]. Several other bacterial strains also appear to only synthesise this lipid when grown in P-deplete medium [5, 37]. These observations suggest a model whereby aminolipids play discrete roles in different bacteria: in some strains the capacity to produce aminolipids has largely been acquired as an adaptation to $\mathrm{P}$ scarcity, whilst in other bacteria they play a more integral role in cell physiology, e.g., for maintaining c-type cytochrome functions [36].

Our analysis of aminolipid synthesis genes in the Tara metagenomes data set provided some support for this hypothesis (Table 2, Suppl. Figures S4, S5). For example, the abundance of ols $B$ showed an overall positive relationship with $\mathrm{N}^{*}$, indicating that it provides a selective advantage in P-deplete conditions. This strong correlation is at least partially explained by the presence of ols $B$ in SAR11 bacteria, which are known to upregulate ornithine lipid production in response to P-stress [5]. On the other hand, the two groups contributing the most to overall ols $B$ abundance, the Rhodobacteraceae and the Rhodospirillales (Suppl. Table S3), showed no significant relationship with $\mathrm{N}^{*}$. Conversely, there was a significant positive correlation between $g l s B$ abundance and $\mathrm{N}^{*}$ in the Tara dataset. However, the abundance of $o l s F$ was not influenced by $\mathrm{N}^{*}$ (Suppl. Figure S4). Unlike OlsB and GlsB, which are primarily found in Alphaproteobacteria, OlsF is the aminolipid synthesis gene most commonly found in Gammaproteobacteria and Bacteroidetes (Fig. 5). A recent lipidomic analysis of one marine Bacteroidetes strain, Dokdonia sp. MED134, showed the presence of several aminolipid classes which comprised a substantial proportion of the lipidome even in P-replete conditions [3]. The role of aminolipids and whether $o l s F$ is responsible for aminolipid synthesis in these marine Bacteroidetes awaits to be determined.

Our genome, metagenome and metatranscriptome analyses showed that the capability to synthesise glutamine lipid appears to be highly conserved in the Rhodobacteraceae whereas the ability to make ornithine lipids is widespread in many ecologically important marine bacteria groups, including the abundant SAR11 clade [5] and the marine Bacteroidetes (Fig. 5). However, only a few studies have reported the detection of aminolipids in the marine environment, and, to the best of our knowledge, no aminolipids other than ornithine lipids have been reported in aquatic ecosystems. One such study, conducted in the Black Sea, detected ornithine lipids in deeper, anoxic water, but not at the surface [6]. The failure to detect ornithine lipids in surface waters is puzzling, given the widespread distribution of $o l s B$ and $o l s F$ in the genomes of sequenced bacteria and marine metagenomes (Fig. 5), and its presence in some strains of the widespread SAR11 clade [5, 11]. At present it is unclear whether the lack of reported aminolipids in marine surface waters reflects shortcomings in the analytical techniques used to detect lipids in these environments, or a genuine lack of these lipids. Our metatranscriptome analysis supports the notion that glutamine lipid biosynthesis occurs 
in marine water columns (Table S1, Figure S3), particularly in members of the numerically abundant and metabolically active pelagic Roseobacter clade [24, 30]. Given the widespread occurrence and expression of aminolipid biosynthesis genes in ecologically important marine bacteria, whether mass spectrometry-based lipidomics techniques have overlooked these compounds certainly warrants further investigation.

Acknowledgements This project has received funding from the European Research Council (ERC) under the European Union's Horizon 2020 research and innovation programme (grant agreement no. 726116). We also thank the Natural Environment Research Council, UK. for a PhD studentship to AFS.

\section{Compliance with ethical standards}

Conflict of interest The authors declare that they have no conflict of interest.

Open Access This article is licensed under a Creative Commons Attribution 4.0 International License, which permits use, sharing, adaptation, distribution and reproduction in any medium or format, as long as you give appropriate credit to the original author(s) and the source, provide a link to the Creative Commons license, and indicate if changes were made. The images or other third party material in this article are included in the article's Creative Commons license, unless indicated otherwise in a credit line to the material. If material is not included in the article's Creative Commons license and your intended use is not permitted by statutory regulation or exceeds the permitted use, you will need to obtain permission directly from the copyright holder. To view a copy of this license, visit http://creativecommons. org/licenses/by/4.0/.

\section{References}

1. Zhang Y-M, Rock CO. Membrane lipid homeostasis in bacteria. Nat Rev Microbiol. 2008;6:222-33.

2. López-Lara IM, Geiger O. Bacterial lipid diversity. Biochim Biophy Acta. 2017;1862:1287-99.

3. Sebastián M, Smith AF, González JM, Fredricks HF, Van Mooy B, Koblížek M, et al. Lipid remodelling is a widespread strategy in marine heterotrophic bacteria upon phosphorus deficiency. ISME J. 2016;10:968-78.

4. Van Mooy BAS, Rocap G, Fredricks HF, Evans CT, Devol AH. Sulfolipids dramatically decrease phosphorus demand by picocyanobacteria in oligotrophic marine environments. Proc Natl Acad Sci USA. 2006;103:8607-12.

5. Carini P, Van Mooy BAS, Thrash JC, White AE, Zhao Y, Campbell EO, et al. SAR11 lipid renovation in response to phosphate starvation. Proc Natl Acad Sci USA. 2015;112:7767-72.

6. Schubotz F, Wakeham SG, Lipp JS, Fredricks HF, Hinrichs K-U. Detection of microbial biomass by intact polar membrane lipid analysis in the water column and surface sediments of the Black Sea. Environ Microbiol. 2009;11:2720-34.

7. Brandsma J, Hopmans EC, Philippart CJM, Veldhuis MJW, Schouten S, Sinninghe Damsté JS. Low temporal variation in the intact polar lipid composition of North Sea coastal marine water reveals limited chemotaxonomic value. Biogeosciences. 2012; 9:1073-84.

8. Kharbush JJ, Allen AE, Moustafa A, Dorrestein PC, Aluwihare LI. Intact polar diacylglycerol biomarker lipids isolated from suspended particulate organic matter accumulating in an ultraoligotrophic water column. Org Geochem. 2016;100:29-41.

9. Geiger O, González-Silva N, López-Lara IM, Sohlenkamp C. Amino acid-containing membrane lipids in bacteria. Prog Lipid Res. 2010;49:46-60.

10. Vences-Guzman MA, Geiger O, Sohlenkamp C. Ornithine lipids and their structural modifications: from $\mathrm{A}$ to $\mathrm{E}$ and beyond. FEMS Microbiol Lett. 2012;335:1-10.

11. Vences-Guzman MA, Guan Z, Escobedo-Hinojosa WI, Bermudez-Barrientos JR, Geiger O, Sohlenkamp C. Discovery of a bifunctional acyltransferase responsible for ornithine lipid synthesis in Serratia proteamaculans. Environ Microbiol. 2015;17:1487-96.

12. Moore EK, Hopmans EC, Rijpstra WIC, Villanueva L, Damste JSS. Elucidation and identification of amino acid containing membrane lipids using liquid chromatography/high-resolution mass spectrometry. Rapid Commun Mass Spectrom. 2016;30:739-50.

13. Wagner-Döbler I, Biebl H. Environmental biology of the marine Roseobacter lineage. Annu Rev Microbiol. 2006;60:255-80.

14. Buchan A, LeCleir GR, Gulvik CA, Gonzalez JM. Master recyclers: features and functions of bacteria associated with phytoplankton blooms. Nat Rev Microbiol. 2014;12:686-98.

15. Schäfer $H$. Isolation of Methylophaga spp. from marine dimethylsulfide-degrading enrichment cultures and identification of polypeptides induced during growth on dimethylsulfide. Appl Environ Microbiol. 2007;73:2580-91.

16. Lidbury IDEA, Murrell JC, Chen Y. Trimethylamine and trimethylamine $N$-oxide are supplementary energy sources for a marine heterotrophic bacterium: implications for marine carbon and nitrogen cycling. ISME J. 2014;9:760-9.

17. Folch J, Lees M, Stanley GHS. A simple method for the isolation and purification of total lipids from animal tissues. J Biol Chem. 1957;226:497-509.

18. Stamatakis A. RAxML version 8: a tool for phylogenetic analysis and post-analysis of large phylogenies. Bioinformatics. 2014; 30:1312-3.

19. Matsen FA, Kodner RB, Armbrust EV. pplacer: linear time maximum likelihood and Bayesian phylogenetic placement of sequences onto a fixed reference tree. BMC Bioinforma. 2010;11:538.

20. Weber T, Deutsch C. Ocean nutrient ratios governed by plankton biogeography. Nature. 2010;467:550-4.

21. Logares R, Sunagawa S, Salazar G, Cornejo-Castillo FM, Ferrera I, Sarmento H, et al. Metagenomic 16S rDNA Illumina tags are a powerful alternative to amplicon sequencing to explore diversity and structure of microbial communities. Environ Microbiol. 2014;16:2659-71.

22. Riekhof WR, Andre C, Benning C. Two enzymes, BtaA and $\mathrm{BtaB}$, are sufficient for betaine lipid biosynthesis in bacteria. Arch Biochem Biophys. 2005;441:96-105.

23. Zhang X, Ferguson-Miller SM, Reid GE. Characterization of ornithine and glutamine lipids extracted from cell membranes of Rhodobacter sphaeroides. J Am Soc Mass Spectrom. 2009;20:198-212.

24. Giebel H-A, Kalhoefer D, Gahl-Janssen R, Choo Y-J, Lee Y, Cho $\mathrm{J}-\mathrm{C}$, et al. Planktomarina temperata gen. nov., sp. nov., belonging to the globally distributed RCA cluster of the marine Roseobacter clade, isolated from the German Wadden Sea. Inter J Sys Evol Microbiol. 2013;63:4207-17.

25. Kang I, Oh H-M, Vergin KL, Giovannoni SJ, Cho J-C. Genome sequence of the marine alphaproteobacterium HTCC2150, assigned to the Roseobacter clade. J Bacteriol. 2010; 192:6315-6.

26. Simon M, Scheuner C, Meier-Kolthoff JP, Brinkhoff T, WagnerDöbler I, Ulbrich M, et al. Phylogenomics of Rhodobacteraceae reveals evolutionary adaptation to marine and non-marine habitats. ISME J. 2017;11:1483-99. 
27. Pesant S, Not F, Picheral M, Kandels-Lewis S, Le Bescot N, Gorsky G, et al. Open science resources for the discovery and analysis of Tara Oceans data. Sci Data. 2015;2:150023.

28. Ricci DP, Silhavy TJ. The Bam machine: a molecular cooper. Biochim Biophy Acta. 2012;1818:1067-84.

29. Durham BP, Grote J, Whittaker KA, Bender SJ, Luo H, Grim SL, et al. Draft genome sequence of marine alphaproteobacterial strain HIMB11, the first cultivated representative of a unique lineage within the Roseobacter clade possessing an unusually small genome. Stand Genom Sci. 2014;9:632-45.

30. Billerbeck S, Wemheuer B, Voget S, Poehlein A, Giebel HA, Brinkhoff $\mathrm{T}$, et al. Biogeography and environmental genomics of the Roseobacter-affiliated pelagic CHAB-I-5 lineage. Nat Microbiol. 2016;1:16063.

31. Gao J-L, Weissenmayer B, Taylor AM, Thomas-Oates J, López IM, Geiger O. Identification of a gene required for the formation of lyso-ornithine lipid, an intermediate in the biosynthesis of ornithine-containing lipids. Mol Microbiol. 2004; 53:1757-70.

32. Weissenmayer B, Gao J-L, Lopez-Lara IM, Geiger O. Identification of a gene required for the biosynthesis of ornithine-derived lipids. Mol Microbiol. 2002;45:721-33.
33. Aygun-Sunar S, Bilaloglu R, Goldfine H, Daldal F. Rhodobacter capsulatus OlsA is a bifunctional enyzme active in both ornithine lipid and phosphatidic acid biosynthesis. J Bacteriol. 2007; 189:8564-74.

34. López-Lara IM, Gao J-L, Soto MJ, Solares-Pérez A, Weissenmayer B, Sohlenkamp C, et al. Phosphorus-free membrane lipids of Sinorhizobium meliloti are not required for the symbiosis with alfalfa but contribute to increased cell yields under phosphoruslimiting conditions of growth. Mol Plant Microbe Interact. 2005;18:973-82.

35. DeChavigny A, Heacock PN, Dowhan W. Sequence and inactivation of the pss gene of Escherichia coli: Phosphatidylethanolamine may not be essential for cell viability. J Biol Chem. 1991; 266:5323-32.

36. Aygun-Sunar S, Mandaci S, Koch HG, Murray IVJ, Goldfine H, Daldal F. Ornithine lipid is required for optimal steady-state amounts of c-type cytochromes in Rhodobacter capsulatus. Mol Microbiol. 2006;61:418-35.

37. Minnikin DE, Abdolrahimzadeh H. The replacement of phosphatidylethanolamine and acidic phospholipids by an ornithineamide lipid and a minor phosphorus-free lipid in Pseudomonas fluorescens NCMB 129. FEBS Lett. 1974;43:257-60. 